\title{
EL PAPEL DE LA PRENSA PEDAGÓGICA EN EL DEPARTAMENTO DE CALDAS (COLOMBIA)
}

\section{The role of the pedagogical press in the department of Caldas (Colombia)}

\author{
Didier Andrés Ospina Osorio \\ Universidad Católica de Manizales \\ Correo-e: daospina@ucm.edu.co \\ Recibido: II de noviembre de 2020 \\ Envío a informantes: is de noviembre de 2020 \\ Aceptación definitiva: Io de enero de 202I
}

RESUMEN: La prensa pedagógica representa una propuesta de investigación emergente en la historia de la educación, reconocida como un escenario abierto de acogida y posibilidad de estudio que ha permitido indagar acerca del papel de la prensa pedagógica en el departamento de Caldas, a través de un estudio documental y bibliográfico que privilegió fuentes primarias y secundarias, en un proceso metodológico basado en la aplicación de tres etapas: Heurística; Doxografía; Etiología, que comprendieron los procesos de recolección, análisis y tratamiento de la información, las cuales permitieron identificar el carácter de la prensa pedagógica en el departamento y la relación temática que presenta esta entre los años i991 y 2020.

Palabras Clave: prensa pedagógica; maestros; educación.

AвstRact: The pedagogical press represents a new emerging research scenario in the history of education in the department of Caldas, recognized as an open scenario of welcome and possibility of study that has allowed us to recognize the role of the pedagogical press in the department of Caldas, a documentary and bibliographic study that privileged primary and secondary sources, in a methodological process based on the application of three stages: Heuristics; Doxography; Etiology, that included the processes of collection, analysis and treatment of the information, which allowed to identify 
the character of the pedagogical press in the department and the thematic relationship that it presents between the years 1991 and 2020.

KEY WORDS: pedagogical press; teachers; education.

\section{Introducción}

$\mathrm{E}$ L ESTUDIO DE LA PRENSA PEDAGógica en el departamento de Caldas se realizó a través de la búsqueda y estudio a profundidad de fuentes primarias y secundarias que permitieron identificar las características de la producción pedagógica de maestros, asociaciones gremiales de educadores, historiadores y demás funcionarios de la educación en este territorio, quienes desde el año i99i han presentado, en las diferentes plataformas físicas y digitales, distintas expresiones comunicativas que han aportado a la reflexión de la educación y el reconocimiento del papel que cumplen los maestros a nivel social y cultural.

Los resultados del estudio destacan una relación temática, determinada en el proceso de revisión documental, que permite reconocer categorías recurrentes, asociadas y emergentes que, a su vez, permiten identificar el carácter reflexivo y pedagógico que presenta la prensa pedagógica producida en el departamento de Caldas entre los años I991 y 2020.

\section{La prensa pedagógica en el departamento de Caldas}

Rastrear las diferentes expresiones comunicativas que permiten conocer la relación cronológica y la orientación temática de la prensa pedagógica producida en el departamento de Caldas permite comprender las categorías de discusión y pronunciación colectiva que la producción escritural de los maestros ha tenido en los últimos 29 años.

Como consecuencia de los derechos y libertades declaradas en el marco constitucional colombiano de I991, diferentes expresiones comunicativas han aparecido a través del tiempo, logrando presentar una palabra viva en cada una de ellas, que posee por lo general un carácter reflexivo y crítico de la sociedad, la educación y la cultura; evocando las diversas miradas de hacer de la educación y de la práctica pedagógica un proyecto de transformación social que valide los rasgos de cambio y posibilidad logrados socialmente con la constituyente.

Desde los diferentes periódicos promovidos por las instituciones educativas, de mucho auge hacia los años 90, hasta la configuración y difusión de diferentes muestras escriturales producidas por los sindicatos municipales y departamentales de profesores, en donde la prensa pedagógica ha tenido un lugar de aceptación entre los actores educativos y la comunidad académica del departamento de Caldas, se reconocen producciones escritas principalmente por maestros, agremiaciones sindicales, directivos y funcionarios administrativos de la educación que han tenido la oportunidad de promover y trascender, desde la palabra escrita, sus pensamientos académicos y sus reflexiones acerca de cómo debería ser la educación en Colombia, evidenciando un llamado insistente y reaccionario que permite inferir las tensiones entre los maestros 
y el sistema educativo colombiano, como una manifestación de reclamo insistente a los gobiernos de turno, ante la necesidad de hacer de la educación un escenario más equitativo y de calidad al servicio de la ciudadanía, así como de la formación de maestros una práctica vocacional y laboral digna para los colombianos.

De allí que el carácter de la prensa pedagógica en el departamento de Caldas durante los últimos veintinueve años no se ha desligado de una fuerte y constante postura política, por el contrario, se acentúa cada vez más al reconocer entre líneas las diferentes posturas políticas que los maestros intentan declarar en cada uno de sus escritos y que permiten, para este caso, reconocer una línea temática relacionada con la presentación del pensamiento pedagógico de los maestros rurales del departamento de Caldas, influenciada desde corrientes de pensamiento y teorías liberadoras como la de Paulo Freire que acompañan y motivan las propuestas y perspectivas recurrentes que los maestros argumentan como posibilidad en la palabra.

Un antecedente histórico lo representa la configuración de la Federación Colombiana de Educadores, la cual incluye más de 23 sindicatos departamentales del país, especialmente desde la celebración del Congreso Nacional de FECODE en 1982, tiempo en el que se creó el Movimiento Pedagógico, el cual motivó y permitió la reflexión de diferentes colectivos de maestros sobre el papel político y cultural de la educación, así como los asuntos asociados a la identidad de los maestros, siendo hasta hoy una parte fundamental en la comprensión de la historia de maestros y maestras que, desde su saber, su práctica pedagógica y su vivencia como mujeres y hombres públicos, han buscado construir una nueva concepción de lucha social desde las aulas y los espacios pedagógicos, como lo afirma Cárdenas (20I2), concebida desde los bordes, distinguiéndose por ser una lucha marcada entre el poder de la política y la cultura que ha contribuido a la reflexión colectiva sobre la imagen social del maestro en las diferentes regiones del país, haciendo que este movimiento no tenga precedente en toda su historia en el territorio colombiano.

Los procesos de tensión política y social marcan la creación de los sindicatos del Magisterio de Educación, los cuales desde la década de los 50 se han proyectado desde la lucha gremial, logrando la conformación específica de la Federación Colombiana de Educadores -FECODE- en 1959, la cual posibilitó la vinculación de profesores de primaria y secundaria, así como la asociación de profesores universitarios -ASPU- bajo la consigna: «Los maestros no son unos apóstoles, son unos explotados». Según lo afirma Cárdenas (20I2), esta consigna significó la ruptura de la imagen del maestro como trabajador asalariado, impuesta por una realidad social que no reconocía su labor y no le garantizaba un salario digno.

Según Rodríguez (1985), la dignificación de la profesión del docente y el mejoramiento de las condiciones laborales del gremio representaron para el imaginario social de los colombianos un cambio importante que marcó la imagen social del maestro en Colombia hasta la década de los 70, cuando el magisterio, después de desplegar una ardua lucha por su reconocimiento profesional, logró hacia el año de i979 la configuración de su primer Estatuto Docente definido legislativamente bajo el Decreto 2277 de 1979.

El hecho de reconocer el carácter laboral de los maestros representó también el reconocimiento del carácter social del maestro otorgado por las condiciones y garantías adquiridas bajo el presente decreto, lo que determinó para quienes, siendo reconocidos como maestros oficiales, comenzaron a gozar de diferentes tipos de condiciones 
y garantías laborales de permanencia, estímulo, ingreso, profesionalización, capacitación y ascenso en el escalafón, que contribuyeron a modificar de alguna forma el «estatus» de los maestros en Colombia.

El registro de la prensa pedagógica en el departamento de Caldas permite reconocer que los maestros y maestras, desde la influencia del Movimiento Pedagógico, han considerado la necesidad de mantener una reflexión colectiva permanente sobre el papel y la responsabilidad del educador, ya no como asalariado, sino como intelectual, como trabajador de la cultura y como ciudadano (Zuluaga, 1987), lo cual ha permitido comprender una variación importante que ha tenido la imagen social del maestro, como un creador de saber y de cultura, percibiéndose como un sujeto que construye y contribuye constantemente a una sociedad que ha reconocido en la educación un escenario de transformación sociocultural.

El rastreo de la prensa pedagógica en el departamento también permite interpretar la responsabilidad política con la que los maestros de la región han motivado la construcción de una imagen social del maestro que lo reconoce como un hombre y mujer públicos, que reconoce su capacidad política en la trascendencia de sus actos, desde lo educativo y lo social, demostrando ser un actor que piensa, siente y vive la educación como un acto emancipador, por tanto, logra percibirse como un constructor de posibilidades ante la lucha constante de hacer de la calidad en la educación una variable determinante para el progreso y transformación social de las comunidades.

La imagen social del maestro, reconocida desde la prensa pedagógica en el departamento de Caldas, identifica a un maestro que a través del tiempo ha evolucionado en su pensamiento y dado un carácter profesional a su labor académica, otorgándole valores agregados a la reflexión pedagógica que ha hecho el gremio.

La selección y revisión documental de la prensa pedagógica sindical del departamento de Caldas producida entre los años I991 y 2020 permitió reconocer un total de I48 publicaciones, algunas de ellas seriadas con publicación recurrente, como el caso de la revista denominada Correo Pedagógico ${ }^{\mathrm{I}}$ y la revista Huellas Femeninas, entre otras publicaciones no recurrentes presentadas ocasionalmente a través de periódicos de tipo escolar. Todas ellas realizadas por maestros, historiadores como Calvo (I963) y Benítez (1994) e investigadores y/o funcionarios de la educación en formatos físicos y digitales asociados a la producción de expresiones comunicativas de corte reflexivo y académico publicadas principalmente entre los años I991 y 2020, algunas de las cuales han mantenido su orientación crítica, declarando abiertamente la intencionalidad informativa y social dirigida a los maestros del departamento, logrando con ello mantener un vínculo comunicativo entre el gremio de los maestros y sus representantes sindicales.

En este sentido, es posible reconocer en la escritura de la prensa pedagógica que fue objeto de estudio categorías recurrentes que aparecen como ejes temáticos que definen el tipo de orientación temática y propositiva que presenta la escritura realizada por los autores, como se hace evidente en la Gráfica i. Categorías recurrentes, que destacan la orientación temática principal que han tenido las publicaciones entre los años I991 y 2020 en el departamento de Caldas, demostrando una tendencia categorial producto de las expresiones comunicativas de los autores.

http://educal.com.co/Noticias/wp-content/uploads/2019/o6/Correo-Pedagogico-34-web.pdf. 
DIDIER ANDRÉS OSPINA OSORIO

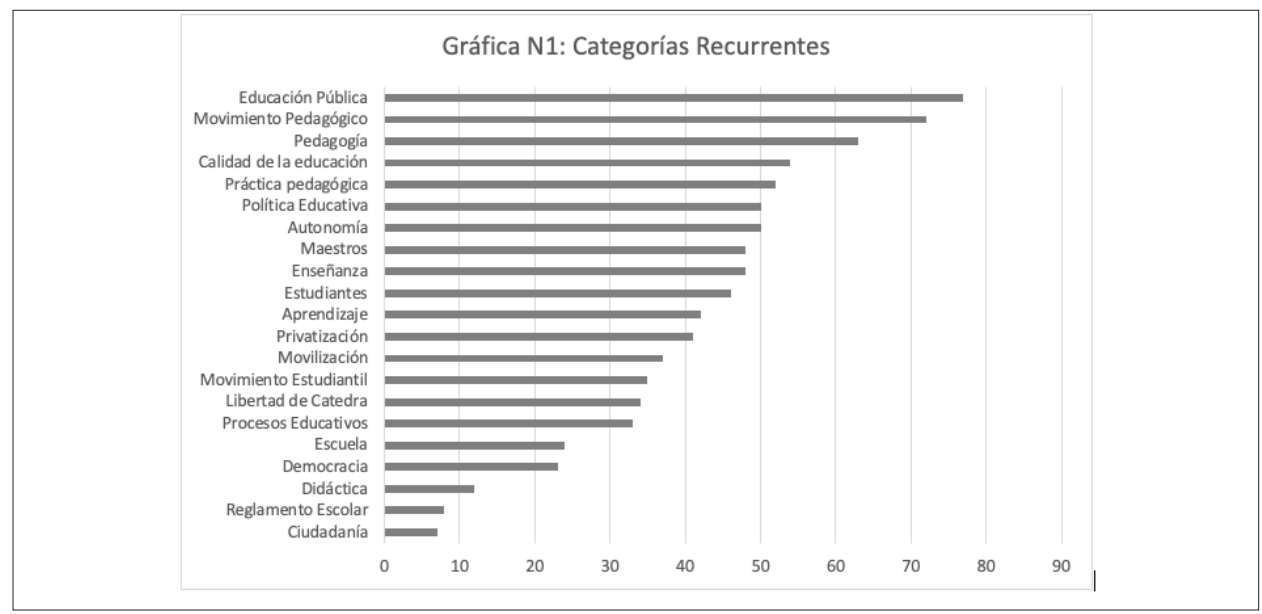

Fuente: Elaboración propia.

Así mismo, la revisión documental permitió el reconocimiento de categorías temáticas asociadas que adicionalmente aparecen en la escritura de la prensa pedagógica y que logran transversalizar la escritura permitiendo, a su vez, reconocer los intereses y sentidos de cada una de las publicaciones, Gráfica 2. Categorías asociadas, que le otorgan sentido y relación a las expresiones comunicativas, destacándose por ser una serie de tendencias teóricas y argumentativas identificadas en los discursos presentados por los autores.

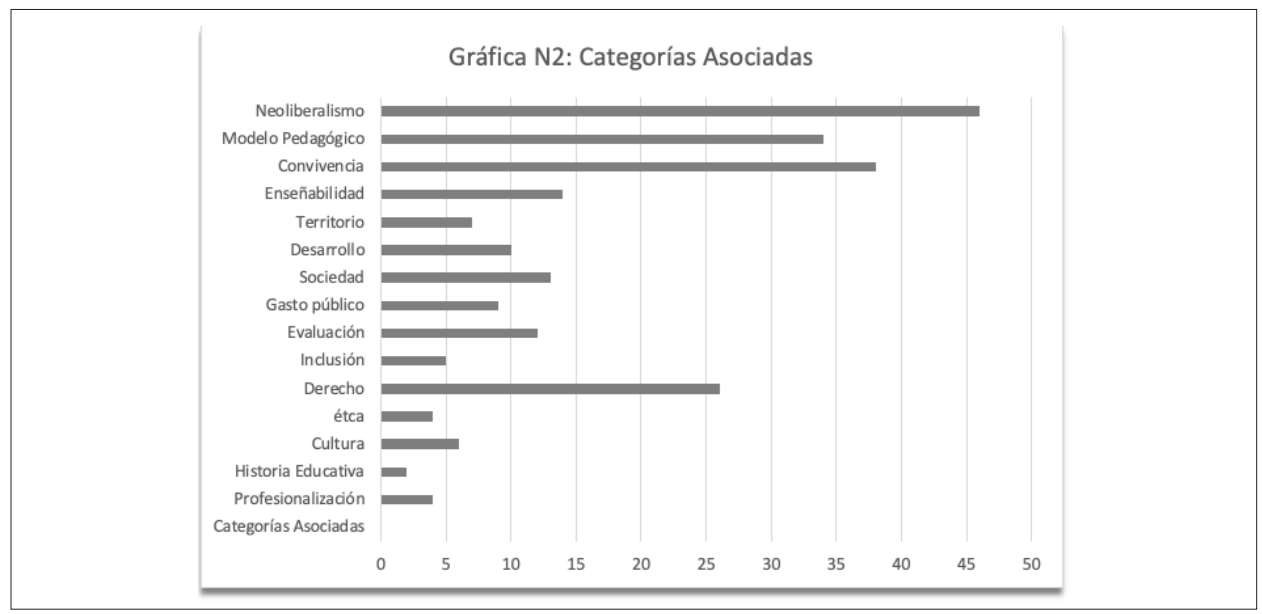

Fuente: Elaboración propia.

La revisión documental permitió precisar las características de una prensa pedagógica abierta, que invita a la reflexión pedagógica y el carácter emancipador de la labor docente desde la integración gremial de los maestros, quienes, a través de la escritura 
y publicación en diferentes plataformas, han reconocido una posibilidad de reflexión pedagógica y a la vez un escenario privilegiado para el logro de la manifestación social de carácter gremial. No obstante, la revisión de la escritura en profundidad permitió hallar categorías emergentes (ver Gráfica 3), las cuales denotan la presencia de discursos asociados a temas que han prevalecido en los últimos io años en el escenario social, cultural, político y académico del país, los cuales han movilizado los discursos presentes en la prensa pedagógica, destacando una gran importancia para la reflexión de los actores educativos del departamento de Caldas.

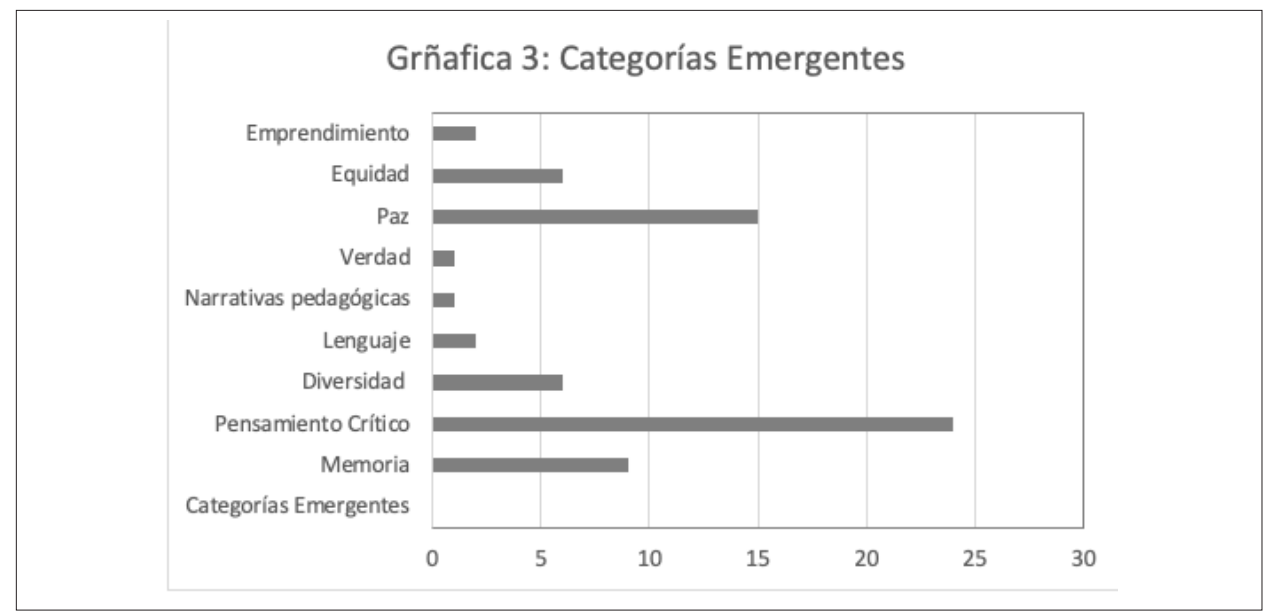

Fuente: Elaboración propia.

La realización de este estudio documental permitió identificar diferentes plataformas escriturales, de ellas, el $58 \%$ físicas y el $42 \%$ digitales, las cuales se reconocen como plataformas de divulgación abierta que han permitido presentar las reflexiones temáticas y gremiales de las comunidades académicas de profesores en el departamento de Caldas, de las cuales el $73 \%$ corresponden a producciones escritas e impresas con divulgación recurrente entre 3 y 6 meses; el 22\% corresponde a publicaciones atemporales presentadas al menos una vez al año, y el $5 \%$ restante obedece a publicaciones que tuvieron difusión ocasional durante la ventana de tiempo en la que tuvo objeto la revisión documental, entre i991 y 2020.

\section{El carácter pedagógico de la prensa en el departamento de Caldas, una oportu- nidad para la investigación}

El programa Prensa Escuela corresponde según el Ministerio de Educación de Colombia a un programa desarrollado por la Asociación Nacional de Diarios -ANDIARIOs- con el apoyo de ese ministerio, que tiene como propósito contribuir al mejoramiento de la calidad de la educación, mediante la implementación de prácticas pedagógicas que incorporen usos innovadores del periódico en el aula. 
De esta manera se ha promovido en Colombia la lectura de la prensa en los ambientes escolares de aprendizaje, así como el desarrollo de habilidades cognitivas de análisis, relación, crítica y memoria, otorgándole a la prensa una función educativa y convirtiendo el periódico en una herramienta para desarrollar habilidades asociadas al fortalecimiento del aprendizaje de los escolares de los niveles de educación básica y media.

El periódico La Patria, como uno de los medios de comunicación más importantes del país y el principal de la región caldense, acogiendo el espíritu pedagógico del programa Prensa Escuela promovido desde el año de 1993 en Colombia, ha desarrollado por más de 20 años en el departamento de Caldas un programa de formación académica que se declara como un «Programa educativo que trabaja de la mano con las instituciones educativas de Caldas para fomentar la lectura en jóvenes y niños, formar corresponsales críticos y crear una comunidad con argumentos» y con ello alcanzar las pretensiones de elevar la calidad de los procesos de aprendizaje de los estudiantes, a través de la relación transversal con diferentes áreas curriculares para permitir el afianzamiento de saberes y experiencias educativas diferenciadoras.

El reconocimiento vivencial de las experiencias pedagógicas resalta los buenos resultados logrados a través del programa, el cual ha construido espacios de participación académica de niños, niñas y jóvenes de las diferentes instituciones educativas del departamento de Caldas.

En este sentido, la revisión de antecedentes permite destacar un vacío en el desarrollo de estudios que permitan comprender el papel de la prensa pedagógica en la formación académica de los escolares, especialmente desde la posibilidad que podría brindar la metodología de sistematización de experiencias, como lo afirma Jara (200I), como un proceso que busque articular la práctica con la teoría, o desde el argumento de Barnechea y González (1994), como una oportunidad de estudio de la prensa pedagógica y su carácter educativo en el departamento de Caldas, reconociendo la escuela como un espacio de convergencia entre las propuestas curriculares y las propuestas de participación y construcción de conocimiento a través del uso de la prensa.

\section{Metodología}

La prensa pedagógica como principal fuente de análisis histórico, según Checa (2002), ha logrado erigirse en el mundo contemporáneo como un ámbito particular y especializado dentro del fenómeno cultural, comunicativo y educativo más amplio que representan la prensa y otros medios de comunicación de masas.

No obstante, como lo afirma Hernández (2015), la prensa pedagógica acoge un amplio espectro de publicaciones educativas y pedagógicas que, para el presente estudio, permitió de manera complementaria la realización de análisis comparado entre las diferentes fuentes primarias y secundarias asociadas a la prensa pedagógica estudiada.

El presente estudio privilegió el desarrollo de un proceso metodológico basado en tres etapas utilizadas en el análisis documental y de fuentes bibliográficas, el cual se empleó con el propósito de orientar el análisis de la prensa pedagógica, implementadose de manera seuencial, con el fin de establecer la relación de sentido entre las categorías de estudio, la recurrencia de cada una de ellas y la posible relación de tendencias asociadas al discurso que presentan las diferentes expresiones comunicativas, así: 
Heurística: Referida a la búsqueda, reconocimiento, localización, recolección, identificación y selección de las fuentes. Este proceso permitió la consolidación de la información bajo la aplicación de criterios de pertinencia y relevancia definidos por el interés específico del estudio.

Doxografía: Este es el momento a través del cual se realizó la ordenación, análisis, clasificación y sistematización de las fuentes ya seleccionadas en desarrollo de la heurística. En esta etapa se estableció un proceso de crítica interna y externa. Cómo lo afirma Suarez (20I8): «La crítica interna atiende y reconoce el carácter racional que resumen los rasgos fundamentales de las fuentes, los conecta con los aspectos secundarios y permite la deducción de nuevas ideas. Por su parte, la crítica externa posibilitó mirar el contexto y resonancia de la fuente» (p. 63).

Etiología: Esta etapa permitió el análisis riguroso de la información recolectada, logrando la interpretación de la misma a partir del reconocimiento de la conexión e interconección de las categorías recurrentes, asociadas y emergentes y la contrucción de sentido existente entre ellas denotando el significado de las expresiones comunicativas, desde la interpretación, la reflexión y la confrontación.

\section{Conclusiones}

En el departamento de Caldas existe una tradición escritural impulsada por las agremiaciones sindicales de profesores, las comunidades académicas representadas por educadores de la región, historiadores y funcionarios del sector educativo que, a través de diferentes plataformas de publicación física y digital, han hecho de la prensa pedagógica una herramienta de expresión y defensa de las libertades de expresión y derecho a la educación concebidos desde el marco constitucional de i99i. El estudio en profundidad de estas fuentes permitió el reconocimiento del papel de la prensa pedagógica en el departamento de Caldas y la oportunidad de conocer su orientación temática, la cual permite validar la influencia de corrientes de pensamiento y teorías liberadoras, desde el reconocimiento de categorías de estudio recurrentes y asociadas presentes en las diferentes expresiones comunicativas.

La prensa pedagógica como objeto de estudio reciente en el departamento de Caldas permite reconocer, entre otros aspectos de interés investigativo, la imagen social del maestro desde los rasgos culturales, sociales y de participación gremial que definen una postura activista y de defensa de los derechos fundamentales, entre ellos la educación, realizada de manera constante por los maestros.

Así mismo, el carácter pedagógico de la prensa en el departamento de Caldas representa un vacío de conocimiento evidenciado a partir de la carencia de estudios que sistematicen la experiencia y validen los aportes que ha realizado la prensa al desarrollo de los procesos educativos en todo el departamento de Caldas.

\section{Bibliografía}

Barnechea, M. y González, M. (1994). «La Sistematización como producción de conocimientos». La Piragua, 9. Taller Permanente de Sistematización-CEAAL-Perú. Consejo de 
Educación de Adultos de América Latina (CEAAL), Santiago. Disponible en http://preval. org/documentos/o0508.pdf. Recuperado el 23 de febrero de 201 .

Benítez, O. M. (1994). La creación cultural en Caldas Vivimos el Mejor Momento de su Inteligencia. ANFORA, 2(3), 5-I4.

Calderón, C. R. M. (20iI). Prensa Escuela: una propuesta de interacción entre juventud, comunicación y escuela. Encuentros, 9(I), 53-68.

Calvo de Vanegas, P. (1963). Riosucio. Manizales: Biblioteca de Autores Caldenses.

Cárdenas Giraldo, M. (2012). El movimiento pedagógico 1982-1998. Bogotá.

Checa Godoy, A. (2002). Historia de la prensa pedagógica en España. Sevilla: Universidad de Sevilla.

Colombia, M. E. N. (1979). Decreto 2277 de 1979, por el cual se adoptan normas sobre el ejercicio de la profesión docente.

Cubillos, C. C. (2014). Impacto del Nuevo Estatuto de Profesionalización en la función docente en Colombia. Análisis de los dos estatutos vigentes: Decreto 2277 de 1979 y Decreto I278 del 2002. Revista Colombiana de Sociología, 37(2), 213-250.

Gómez Duarte, R. (1997). Programa Prensa-Escuela Colombiano. Comunicar, 8, 179. Accessed 9 Nov. 2020.

Hernández DíAz, J. M. a (ed.). (2015). La prensa de los escolares y estudiantes: su contribución al patrimonio histórico educativo. Ediciones Universidad de Salamanca.

Jara, O. (200I). Dilemas y Desafíos de la Sistematización de experiencias. Presentación en el Seminario asocam: Agricultura Sostenible Campesina de Montaña, organizado por Intercooperation Cochabamba, Bolivia. Publicado por el CEP Centro de Estudios y Publicaciones Alforja. Disponible en http://www.redalforja.net/redalforja/images/stories/PDF-DOCS/ Sistematizacion_experiencias.PDF. Recuperado el is de agosto de 2010.

JARA, O. (no registra fecha). La sistematización de experiencias y las corrientes innovadoras del pensamiento latinoamericano - una aproximación bistórica. Disponible en http://www.infoandina.org/system/files/recursos/oscar_jara_sistematizacion_y_corrientes_innovadoras. pdf. Recuperado el 20 de agosto de 20 Io.

Lombianos. Educación y Cultura (13). Bogotá.

Macia, M. B. (2009). La profesionalización docente en Colombia. Revista Colombiana de Sociología, 32(2), III-I3I.

Rodríguez Bello, W. (20r8). El Imaginario Social de la Dirigencia Sindical y su Incidencia en la Construcción de Identidad Colectiva. Asociación Distrital de Educadores ADE 2012-2017.

Suárez, P. T. P.; Araméndiz, M. S. y Mendoza, E. M. La Instrucción en Primera Plana: El Papel Pedagógico y Civilizador de la Prensa Educativa en Manizales 1905-1920. En VII Simposio Colombiano de Historia Local y Regional Asociación Colombiana de Historia (p. 63).

Zuluaga, O. L. (1987). El Movimiento Pedagógico: taller de los maestros colombianos. Educación y Cultura, (I3). 
